\title{
Algorithm and Scheme for Spectrum Mobility in Cognitive Radio Oriented Wireless Network
}

\author{
Mardeni Roslee, Senior Member, IACSIT, K. Anuar, M. Y. Alias, H. Mohamad, N. Ramli, and K. \\ Ismail
}

\begin{abstract}
We develop a new algorithm and performed a measurement for spectrum mobility in cognitive radio network. The operating frequency range is $2.4 \mathrm{GHz}$ to $2.5 \mathrm{GHz}$. An algorithm for spectrum handoff developed is able to make the cognitive user to produce efficient decision to return the spectrum to demanded licensed user. By using our proposed scheme, the cognitive user able to adjust its transmission power with sufficient protection to licensed user instead of carrying out spectrum handoff and it is found working nicely. In this project, measurement and simulation were implemented and provide agreement around $89 \%$ to $92 \%$. The purpose is to analyze a new architecture for spectrum mobility and handoff with a sufficient level of Quality of Service guarantee by Internet System Provider for more energy efficient communications. Energy-efficiency of mobile networks not only reduces environmental impact, it also cuts network costs and helps to make communication more affordable for everyone.
\end{abstract}

Index Term-Spectrum mobility, handoff, cognitive radio.

\section{INTRODUCTION}

Cognitive radio devices able to sense their environment, adapt to different conditions, and incorporate long term learning. Integrating situational awareness of the wireless spectrum, power grid usage, and the needs of the systems users with the power control of base station devices opens up a new world of cognitive power control. In this project, it is expected that the contributions is towards the cognitive radio and dynamic spectrum access, and applicationoriented contributions dealing with new technology of system designs. Finding new efficient-energy solutions for spectrum mobility and handoff decision also helps spread access to communications by opening up more options for the sitting of radio sites in a sustainable, low-impact way, and reducing overall total cost of ownership.

\section{LITERATURE REVIEW}

Currently, inefficient use of spectrum data volume increases approximately by a factor of 10 every 5 years,

Manuscript received May 4, 2014; revised September 15, 2014. The research is fully sponsored under government grant, science fund, ministry of science and technology (01-02-01-SF0191).

Mardeni Roslee, K. Anuar, K. Ismail, and M. Y. Alias are with the Faculty of Engineering, University of Multimedia, Jalan Multimedia, 63100 Cyberjaya, Selangor, Malaysia (e-mail: mardeni.roslee@mmu.edu.my, khairil@mmu.edu.my, yusoff@mmu.edu.my).

H. Mohamad and N. Ramli are with the Wireless Communications, MIMOS Berhad, Kuala Lumpur, Malaysia (e-mail: hafizal.mohamad@mimos.my, nordin.ramli@mimos.my). which corresponds to an increase of the associated energy consumption by approximately $16-20 \%$ per year. There are $3 \%$ of the world-wide energy is consumed by telecommunication system infrastructure that causes about $2 \%$ of the world-wide $\mathrm{CO}_{2}$ emissions, which is comparable to the world-wide $\mathrm{CO}_{2}$ emissions by airplanes or one quarter of the world-wide $\mathrm{CO}_{2}$ emissions by cars [1].

In current spectrum technology, there has been a growing interest in cognitive radio oriented network where the technology that promises to overcome the problem of spectrum mobility resulting from the current approach of fixed spectrum allocation. At the device level, each cognitive radio is able to learn/understand the radio environment and to dynamically adapt its operating parameters to make best use of the available spectrum. This is achieved by equipping radio devices with enabling capabilities such as spectrum sensing, adaptive transmission, and software reconfigurability. At the system level, cognitive radio networks, formed by cognitive radio devices, is to make better usage of available spectrum to achieve higher end-to-end quality of service, e.g., in throughput and/or delay performance [2]. However, developing an algorithm and scheme of fully-functioning cognitive radio network can be challenging.

In the traditional management of licensed spectrum, users usually pay and have the exclusive access of spectrum with a minimum level of Quality of Service (QoS) guarantee. On one hand, the spectrum is getting more and more crowded as the number of wireless devices increases drastically. However, on the other hand, the utilization of spectrum at any given time is low. It can be seen that a lot of spectrum bands are not fully utilized. Therefore, it would be efficient to allow unlicensed users to share spectrum with licensed users by using a unoccupied frequency band [2] .

Cognitive Radio technology is developed to utilize these white spaces intelligently. FCC Spectrum Policy Task Force published a new spectrum management policy, open access or license exempted model to allow unlicensed user or secondary user to use the unoccupied spectrum, known as opportunistic spectrum [3]. Seen as the secondary user, the cognitive radio (CR) must avoid interfering with primary user (PU), that is, licensed user, while sharing the licensed band with the PU. Therefore, cognitive radio needs to sense the spectrum to detect the existence of PU, identify the white spaces of spectrum, and adapt its transmission to one of the white spaces to avoid interfering with PU [4], [5]. There would be are more vacant channels available that can causes a world-wide energy waste.

Detecting the vacant bands of the spectrum is the crucial initial step but very crucial in Cognitive Radio technology. 
There are three major cognitive radio network techniques that could be used to detect the existence of PU which are matched filtering, energy detection, and cyclostationary feature detection [6]. Among those, energy detector has been used widely due to its simplicity and easy implementation. However as a radio device, a single CR may suffer severe shadowing or multipath fading with respect to primary transmitter so that it cannot detect the existence of PU even in its vicinities. In addition, there exists a hidden-node problem, in which a CR may be too far from the PU to detect the existence, but close to the primary receiver to interfere with the reception if transmited [7]-[9]. Due to these challenges, an efficient algorithm and scheme are required in cognitive radio oriented wireless network for certain level of Quality of Service (QoS). A multiple cognitive radios cooperate to reach an optimal global decision by exchanging and combining individual local sensing results. Allowing multiple users to cooperate, cooperative sensing can increase the detection probability, reduce the detection time, and achieve the diversity gain.

\section{METhODOLOGY}

The project objective is divided into 2 paths which are algorithm development and experimental design. The operating frequency range is 2.4 to $2.5 \mathrm{GHz}$.

\section{A. Proposed Spectrum Handoff Algorithm}

In algorithm development, this paper focuses on spectrum handoff where cognitive user can returns the spectrum to demanded licensed user. Besides, the cognitive user can chooses to adjust its transmission power with sufficient protection to licensed user instead of carry out spectrum handoff. Using our proposed scheme, cognitive user switches between the bands if and only if it is unable to modify its transmission power within the tolerable limits. Therefore, this paper aims to clearly describe the concepts of cognitive radio and fuzzy logic, and the implementation of proposed algorithm. MATLAB software simulations were carried out and the results show the promising benefit of using our scheme in optimizing the power consumption and spectrum handoff rate of cognitive radio.

In this work, spectrum handoff algorithm is proposed and illustrated in the form of a flow chart as shown Fig. 1 below. Our spectrum handoff algorithm focuses on the idea instead of returning the frequency band back to reclaim primary user (PU) and transferring to other idle bands, secondary user (SU) should try to adjust its transmission power.

As illustrated in Fig. 1, when the time the algorithm is activated, the aspect of SU needs to compare values of the power transmitted from the PU and the power it can receives from PU transmission. This comparison will act as the reference to measure the distance between the users, and calculate the power at which SU should transmit. SU changes its power transmitted accordingly to ensure no interference causing to neighbor PUs. It is worth to mention that sometimes the power transmitted by SU can be reduced to avoid redundant spectrum handoff. However, the Shannon-Hartley theorem mentioned that if the power transmitted of SU becomes very low, the rate of transmission will drop as (1).

$$
R_{S U}=B_{C} \log _{2}\left(1+\frac{P_{S U}}{N_{0}}\right)
$$

where $R_{S U}$ is the $\mathrm{SU}$ transmission rate, $B_{C}$ is the channel bandwidth, $P_{S U}$ is SU transmit power and $N_{0}$ is the thermal noise. In other words, QoS becomes terrible and the spectrum handoff will be the only option here. Therefore, our algorithm is designed to reduce the interference as low as possible, and relying on QoS parameter, system will decide whether the spectrum handoff should take place.

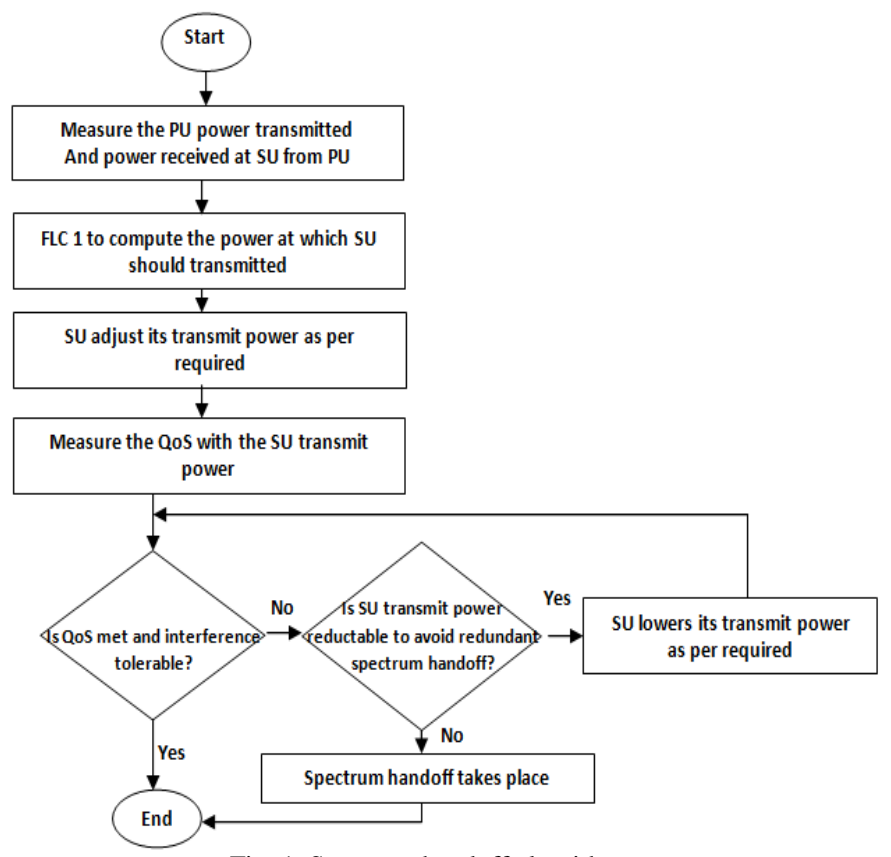

Fig. 1. Spectrum handoff algorithm.

\section{B. Implementation of Proposed Algorithm}

The spectrum handoff algorithm is implemented in a hierarchical model where two Fuzzy Logic Controller, FLCs are used as shown in Fig. 2. The first FLC is in charge of measuring the distance between users, and controls SU transmit power, $P_{s u}$; while the second FLC is in charge of making decision if handoffs to another frequency band is required or not. The second controller consists of two outputs which are handoff power, $P_{\text {handoff }}$ and power modification decision, $M O D_{P s u}$. The first output also defined as $P_{\text {handoff }}$ indicates whether a spectrum handoff is needed to carry out. The second output also defined as $M O D_{P s u}$ indicates whether the power transmitted by SU is needed to adjust. The actual actions of FLCs are explained in detail at the following sections.

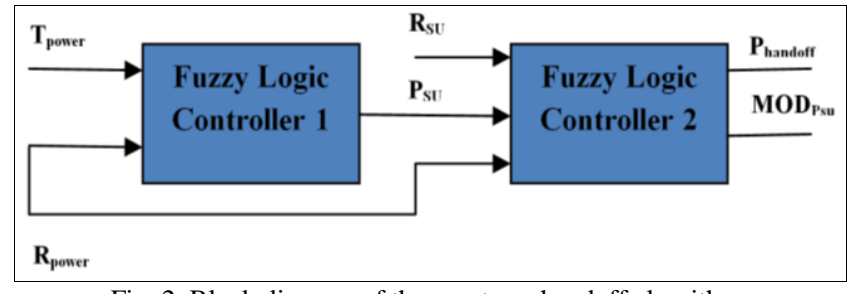

Fig. 2. Block diagram of the spectrum handoff algorithm.

\section{Type 1 Simulation Results}

Fig. 3 to Fig. 5 illustrate the graph of SU's transmission 
power, spectrum handoff decision and power modification decision versus power received by SU from PU. The graphs in the Fig. 3 to Fig. 5 consist of three different colours and pattern of lines, where each of them represents as a user with a value within the range of the $T_{\text {power }}$ : The red colour line represents $T_{\text {power }}=-14 \mathrm{dBm}$ (low power scale); green colour line represents $T_{\text {power }}=0 \mathrm{dBm}$ (medium power scale); blue colour line represents $T_{\text {power }}=14 \mathrm{dBm}$ (high power scale). Both of graphs in the figures illustrate that the distance between SU to PU is change from far to near when the $R_{\text {power }}$ is increased.

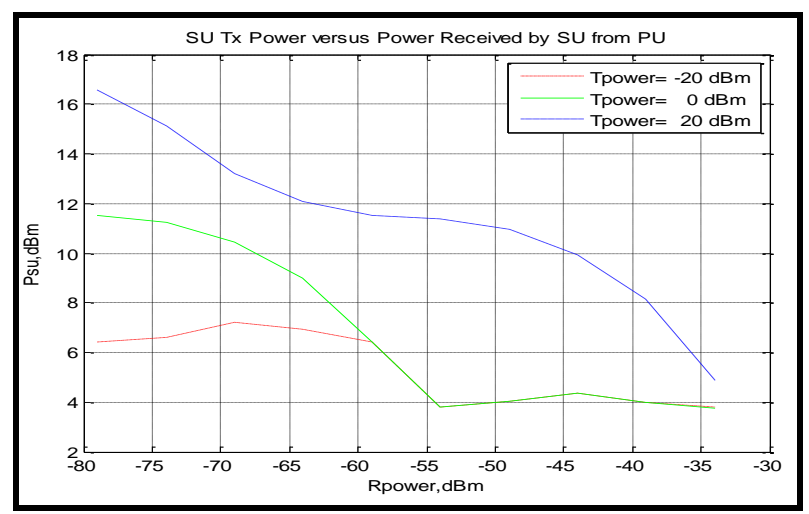

Fig. 3. Graph of $\mathrm{P}_{\mathrm{SU}}$ vs. $\mathrm{R}_{\text {power }}$.

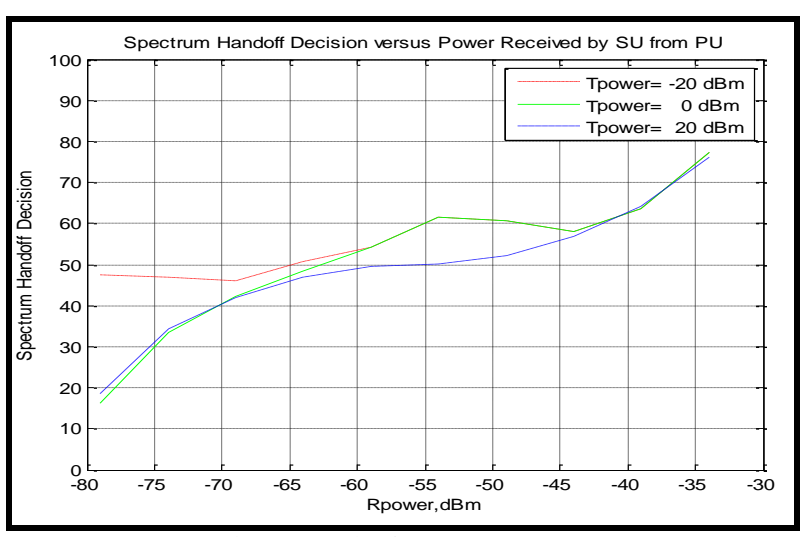

Fig. 4. Graph of $P_{\text {handoff }}$ vs. $R_{\text {power }}$.

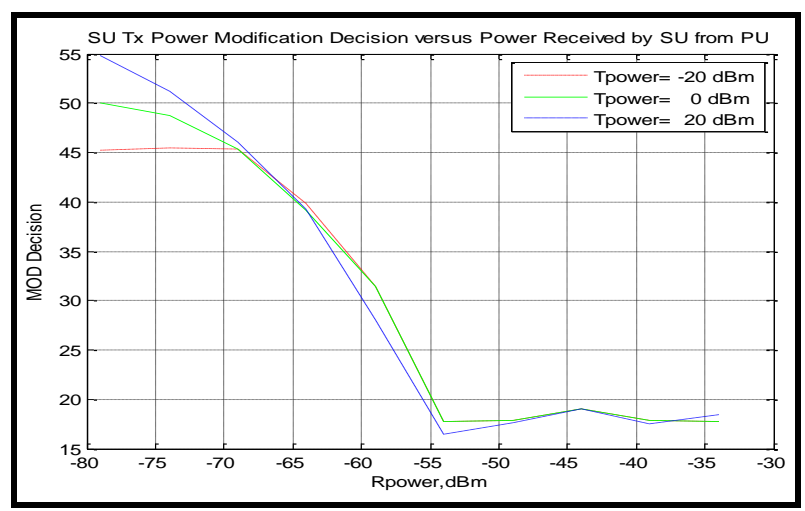

Fig. 5. Graph of $\mathrm{MOD}_{\mathrm{Psu}}$ vs. $\mathrm{R}_{\text {power }}$

Fig. 3 represents the graph of subscriber user power against received power. It can be investigated that when the value of $R_{\text {power }}$ is increasing, the value of $P_{S U}$ is decreasing because perception of SU moving closer to PU. Thus, SU should reduce its transmission power to avoid interference to PU. The value of $\mathrm{P}_{\mathrm{SU}}$ of the blue line in Fig. 3 shows highest, followed by green and red lines because the blue line is far to the PU when compared to green and red lines.
In other words, the interference caused by the blue line to the PU is less possible.

Fig. 4 represents the graph of handoff power against received power. It found that when the value of the $R_{\text {power }}$ is increasing, the value of $P_{\text {handoff }}$ is increasing. This is due to the perception of SU moving closer to PU. Therefore, SU is required to perform a spectrum handoff when the value of $P_{\text {handoff }}$ exceeds certain limit. The red line in Fig. 4 shows highest chance to perform handoff, followed by green and blue lines because it is near to the PU when compared to green and blue lines. It also shows that the results are within the acceptable range [3]-[4]. The graph in Fig. 5 represents the power modification decision against received power. When the value of the $R_{\text {power }}$ is increasing, the value of $M O D_{P s u}$ is decreasing because perception of SU moving closer to PU. SU is recommended by the system on reducing its transmission power, $P_{S U}$. The power reducing method gives the chance for SU to avoid a spectrum handoff when the condition is met. It means the QoS of SU transmission is acceptable. The work has been extended into experimental design in order to analyze more the received power in spectrum.

\section{Experimental in the Lab}

In measurement path, the mobile testbed architecture is shown in Fig. 6. One Universal Software Radio Peripheral, USRP has been configured to be the primary users and other to be the secondary users. They transmit packets of random content at fixed carrier frequencies. Like primary users, the USRPs do not transmit data all the time and are programmed to transmit data at random intervals and for random lengths of time. The actual measurement setup is shown in Fig. 7.

The secondary users are associated with an ID number which helps them uniquely identify each other. Since they have no licensed spectrum, they opportunistically use any vacant frequency bands. The secondary users are configured to perform the following tasks: 1) Decide if a given frequency band is currently occupied or not; 2) Change the transmitting and receiving parameters at any given time; and 3) Make sure that its operation does not interfere with primary users. Thus, the USRPs acting like secondary users are programmed such that their structures and parameters change according to the channel conditions. There is also a laptop are used which acts as a monitor, providing the ground truth of the state of spectrum utilization.

USRPs are software defined radios which are connected to a computer terminal or laptop via a USB cable. To gain all the functionalities of a software defined radio, the USRPs work on the GNU radio platform. GNU radio software is an open source toolkit which allows the construction of radios where all hardware related problems like designing and building the required circuits are converted to software issues. The laptop is used to view the state of spectrum utilization.

All the USRPs are connected to laptop where LINUX is running to host the GNU radio software. The GNU radio software comes with programs which can be used for packet transmission and reception and has many reconfigurable options like carrier frequency modulation type, data rate, packet length etc. To emulate a cognitive radio network, the USRPs are configured to take different roles. In this section, 
provide the algorithms and software configuration details that achieve these roles on the USRPs.

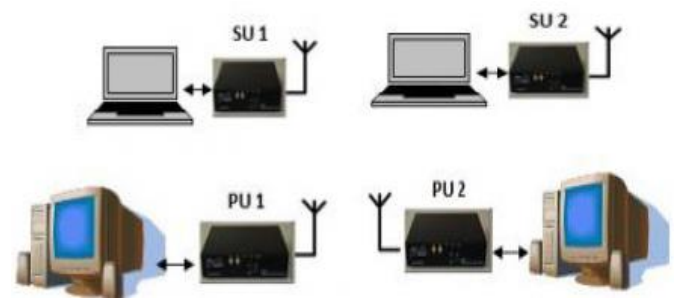

Fig. 6. Measurement block diagram

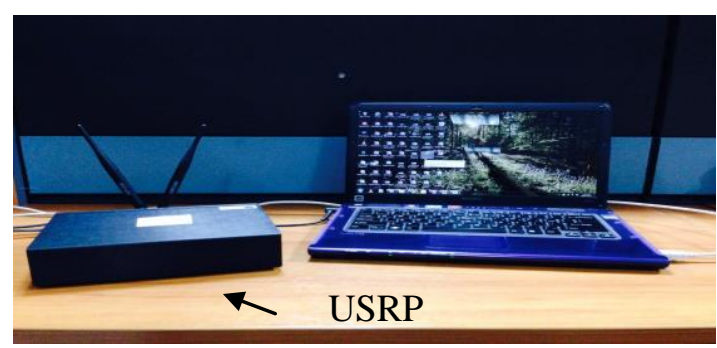

Fig. 7. Measurement setup

\section{EXPERIMENTAL RESULTS}

In this section we evaluate the performance of our testbed using two main criterias. The first one is the ability to sense a piece of spectrum and decide the unoccupied channels, i.e., spectrum sensing and second is to check the ability of the whole system demonstrating dynamic spectrum access. Various operating parameters of the cognitive radio network are provided.

As mentioned in previous section, the USRP are used in our system have the capability to operate on both $2.4 \mathrm{GHz}$ and $2.5 \mathrm{GHz}$ range. The $2.4 \mathrm{GHz}$ spectrum is chosen to avoid interference from the Wi-Fi network of the university faculty operating in range. To make the primary user signals different from the secondary users, the primary users transmit different modulated packets compared with the secondary users.

This experiment was conducted in order to observe the spectrum utilization of the proposed range as well as to compare with the proposed algorithm. By using the usrp_spectrum_sense.py, Fig. 8-Fig. 13 spectrums are obtained.

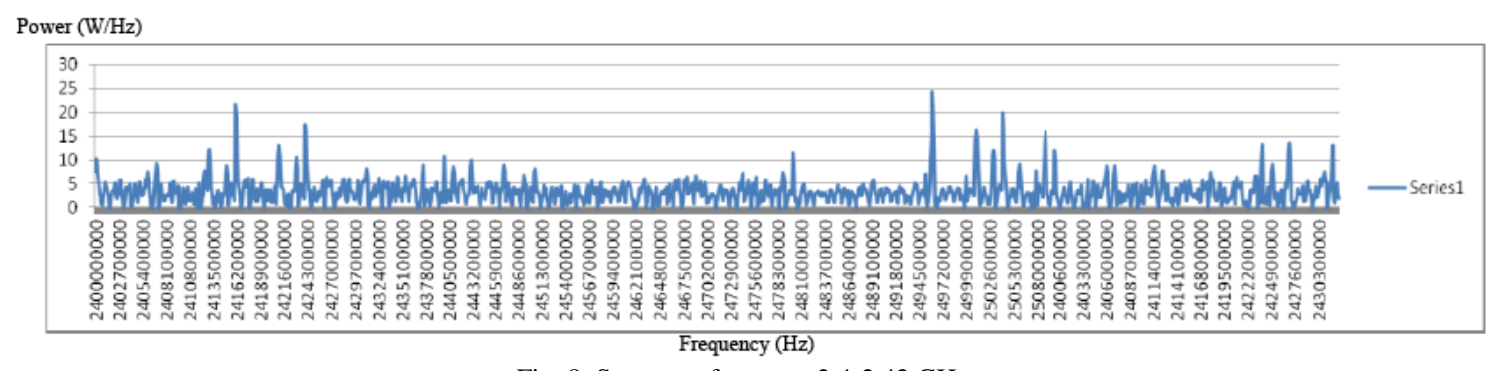

Fig. 8. Spectrum for range $2.4-2.43 \mathrm{GHz}$.

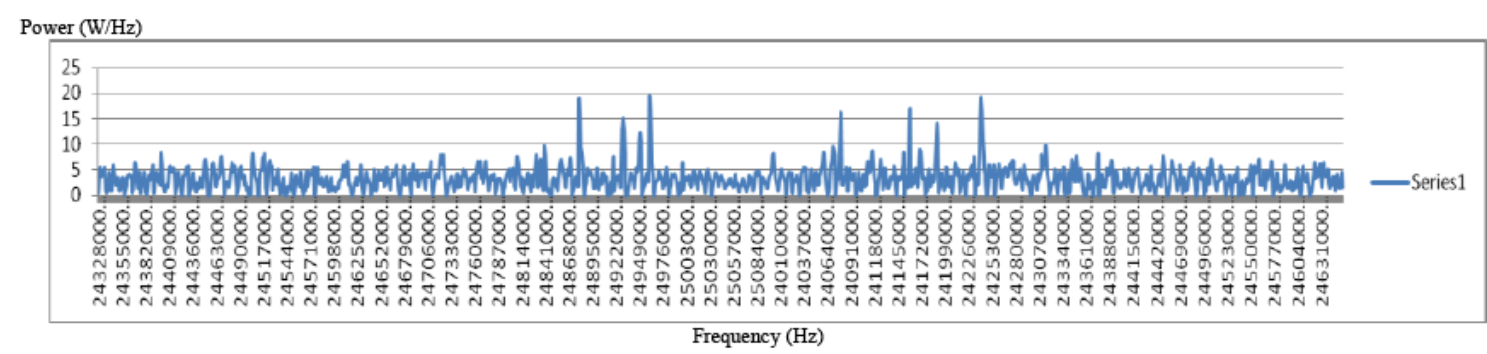

Fig. 9. Spectrum for range $2.43-2.46 \mathrm{GHz}$.

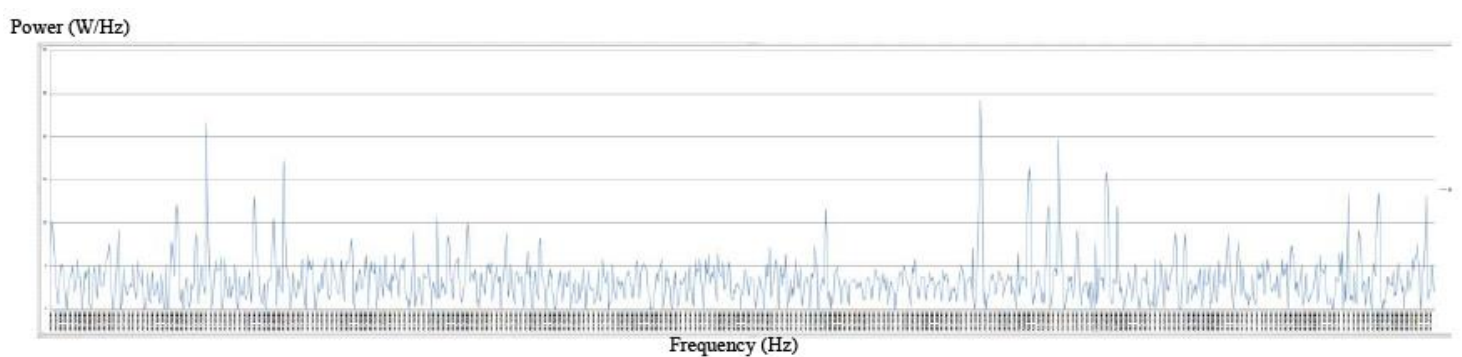

Fig. 10. Snapshot of the spectrum using USRP for range $2.4-2.5 \mathrm{GHz}$.

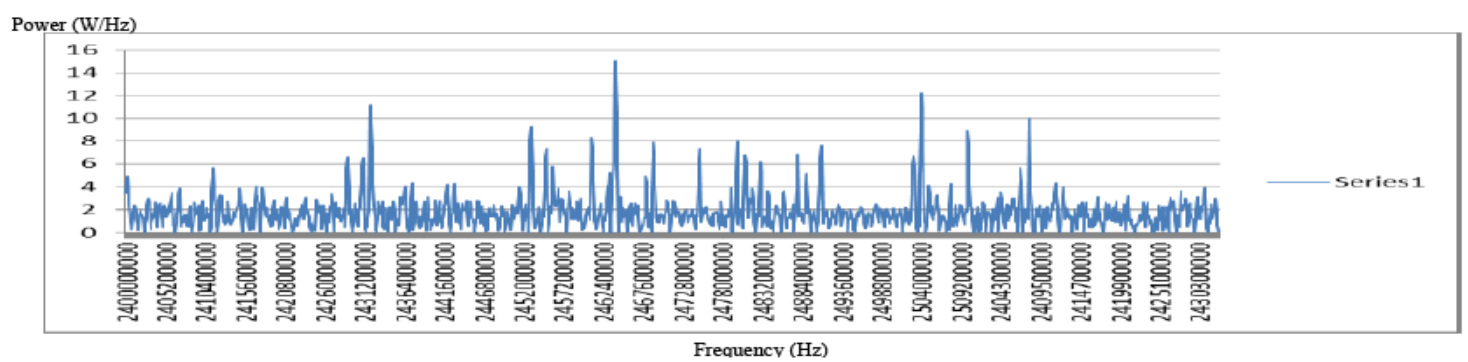

Fig. 11. Spectrum range for $2.4-2.43 \mathrm{GHz}$. 


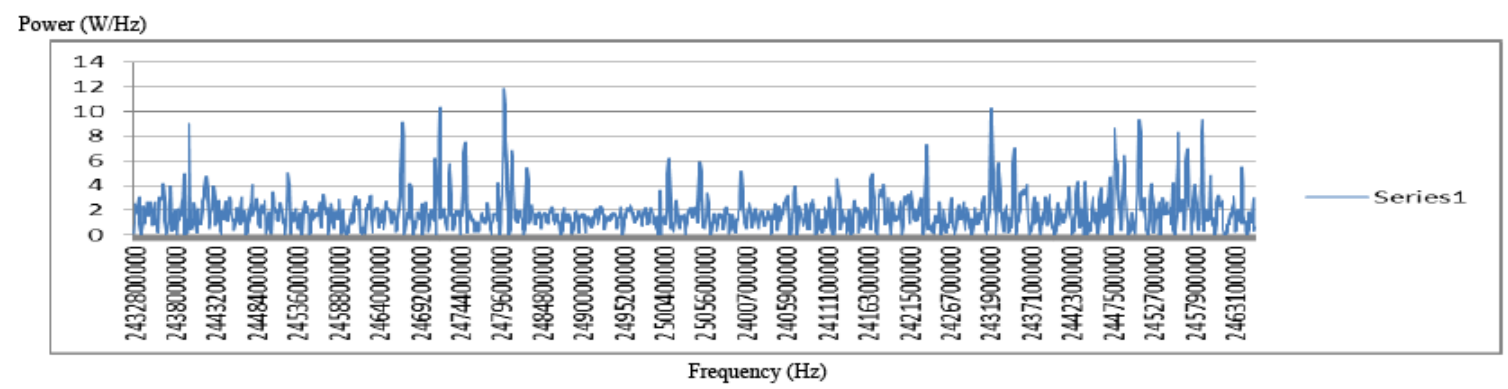

Fig. 12. Spectrum range for $2.43-2.46 \mathrm{GHz}$

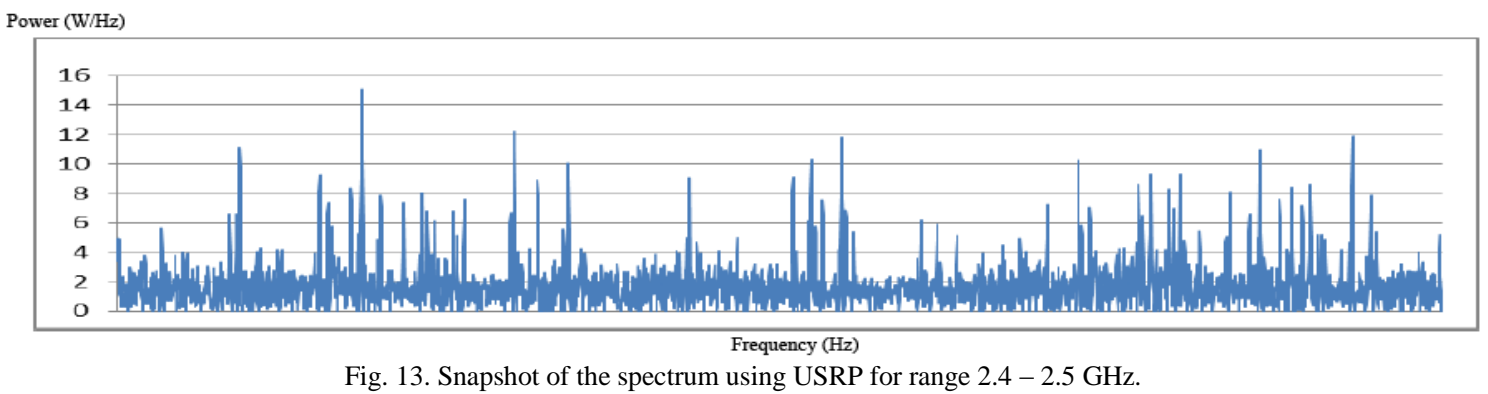

The experiment was done at different hours of the day to analyse the spectrum utilization. The results of measurement in the first phase can be seen in Fig. 8-Fig. 10 whereas data obtained in the second phase can be seen in Fig. 11-Fig. 13. The noise level in Fig. 8-Fig.10 is around $5 \mathrm{~W} / \mathrm{Hz}$. Assuming the spikes in the spectrum are signals, it is found that some spectrum range are underutilized or holes are existed [10]. Figure 8 shows the spectrum collected for range $2.4-2.43 \mathrm{GHz}$ while Fig. 9 shows the spectrum collected for range $2.43-2.46 \mathrm{GHz}$. Fig. 10 is a combination of the first two spectrums. The $x$-axis represents the frequency with unit Hertz, while the $y$-axis represents the received power in $\mathrm{W} / \mathrm{Hz}$. The noise level in Fig. 11-Fig. 13 appears to be around $3 \mathrm{~W} / \mathrm{Hz}$ which is lower than the measurement conducted in the morning. It is found that the spectrum range is fairly utilized or moderately used [11], which is different from findings presented in Fig. 8-Fig. 10. Through this measurement, we are able to get power spectral densities of the existing signals. Thus, we can deduce many features of a spectrum like how good a channel is in terms of noise, the average power in a channel, the peak power observed, etc.

From these experimental results, it then compared with the proposed algorithm results as described before for verification. The observation is implemented for SU's transmission power within the range of low power scale, $20 \mathrm{dBm}$, medium power scale, $0 \mathrm{dBm}$ and high power scale, $20 \mathrm{dBm}$. From the experiment, it shows that the distance between SU to PU, as in Fig. 6, is change from far to near when the received power is increased. Another finding is when the value of received power is increasing, the value of secondary user power is decreasing because perception of SU moving closer to PU. The mean error for each point of secondary user power is calculated using equation (2) in order to observe the agreement between measurement and simulation. The percentage of agreement between the measurement and simulation are $92 \%, 91 \%$ and $89 \%$ for low power scale, medium power scale, and high power scale, respectively. From this result, it able to verify the spectrum handoff decision and power modification decision as well.
Besides, it also found that different sensing algorithms are realized on the testbed to demonstrate their effectiveness in identifying spectrum holes. The system is extended to incorporate collaborative spectrum sensing amongst the secondary users. Experimental results show that the reconfigurability of the secondary users allows them to adapt to the channel occupancy states. Although, the current testbed is focused on spectrum mobility and dynamic spectrum access, it has the ability to carry out extensive research in all fields of the cognitive radio. Seen as the secondary user, the cognitive radio (CR) must avoid interfering with primary user (PU), that is, licensed user, while sharing the licensed band with the PU. Therefore, cognitive radio needs to sense the spectrum to detect the existence of PU, identify the holes of spectrum, and adapt its transmission to one of the white spaces to avoid interfering with PU.

\section{CONCLUSION}

In this work, new algorithm has been proposed and the simulation results are performed by using MATLAB software. The performance comparison has been made between measurement and simulation and provides agreement around $89 \%$ to $92 \%$. From the observation, it is found that the average SU transmission power of our designed algorithm is consumed more than the other algorithm when the distance between PU and $\mathrm{SU}$ is constantly far over certain periods. As a result, its signaling cost is higher than other algorithm. On other hand, the processing cost of our designed algorithm is lower than other algorithms because it still guarantees a low spectrum handoff rate, where it is sufficient to convince that the spectrum mobility function is performed efficiently. The work has been extended to the experimental in the lab to verify the proposed algorithm, where a cognitive radio testbed built on USRPs. By configuring the USRPs to play different roles, we are successful in implementing a basic wireless communication network with cognitive abilities where different sensing algorithms are realized on the testbed to 
demonstrate their effectiveness in identifying spectrum holes.

\section{REFERENCES}

[1] B. Le, T. W. Rondeau, and C. W. Bostian, "Cognitive radio realities," Wireless Communications and Mobile Computing, vol. 7, 2007.

[2] H. Zhang, "Cognitive radio for green communications and green spectrum," Chinacom, Hangzhou, China, 2008.

[3] D. Niyato, E. Hossain, and Z. Han, "Dynamics of multiple-seller and multiple-buyer spectrum trading in cognitive radio networks: A game theoretic modeling approach," IEEE Transactions on Mobile Computing, vol. 8, no. 8, pp. 1009-1022, August 2009

[4] M. A. Sturza and F. Ghazvinian, "White spaces engineering study: Can cognitive radio technology operating in the TV white spaces completely protect licensed TV broadcasting?" New America Foundation Wireless Future Program, Working Paper no. 16, January 2007.

[5] K. Watanabe, K. Ishibashi, and R. Kohno, "Performance of cognitive radio technologies in the presence of primary radio systems," in Proc. the 18th Annual IEEE International Symposium on Personal, Indoor and Mobile Radio Communications, September 2007.

[6] D. Niyato, E. Hossain, and Z. Han, "Dynamic spectrum access in IEEE 802.22-based cognitive wireless networks: A game theoretic model for competitive spectrum bidding and pricing," IEEE Wireless Communications, vol. 16, no. 2, pp. 16-23, April 2009.

[7] E. C. Y. Peh, Y.-C. Liang, and Y. L. Guan, "Optimization of cooperative sensing in cognitive radio networks: a sensing-throughput tradeoff view," in Proc. the IEEE International Conference on Communications, June 2009, pp. 1-5.

[8] G. Ganesan and Y. Li, "Cooperative spectrum sensing in cognitive radio networks," in Proc. the 1st IEEE International Symposium on New Frontiers in Dynamic Spectrum Access Networks, November 2007, pp. 137-143.

[9] L. Duan, J. Huang, and B. Shou, "Competition with dynamic spectrum leasing," IEEE DySPAN, April 2010.

[10] H. Stark and J. W. Woods, Probability And Random Processes with Application to Signal Processing Third Edition, Prentice Hall, 2002, pp. 66-75.

[11] R. Rao, Q. Cheng, A. Kelkar, and D. Chaudhari, "Cooperative cognitive radio network testbed," ICaST, June 2011.

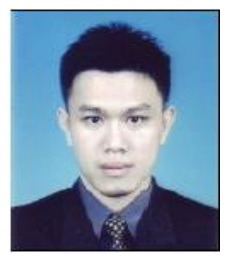

Mardeni Roslee received his bachelor degree in electronic (Hons) from UKM, and master's degree in telecommunication and $\mathrm{PhD}$ degree in communication network engineering from UPM, respectively. He is a chartered engineer awarded from UK. He has been a faculty member at the Multimedia University, Malaysia from 2008 until now. He is a senior member of IACSIT, Member of IEEE and IET. His current research interests are wireless and mobile communication with radar system. He has published over 40 international journal and conference papers. He has been involved in organizing a number of conferences including APWBF09, AFICT09, PIERS12, ISTT12 and MICC13. He has won some research competitions including ITEX05, ITEX13 and PRPI09. He has 12 graduated postgraduate students in Masters and $\mathrm{PhD}$. He has research grant amounting USD 273, 000.

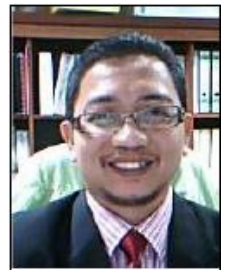

Mohamad Yusoff Alias received his B.Sc.Eng. (E.E.) degree from Michigan, USA and PhD degree from Southampton, UK. He has been a faculty member at Multimedia University, Malaysia from 1998 and as an associate professor since 2010. He is a senior member of IEEE. His current research interests are OFDM, femtocell and Hetnet. He serves as deputy dean of Institute of Postgraduate Studies and Multimedia University. $\mathrm{He}$ was an honorary academic visitor to University of Manchester in year 2011.

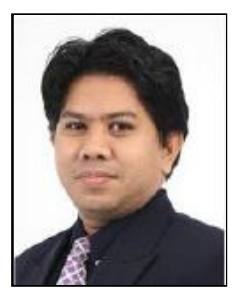

Hafizal Mohamad received the B.Eng. degree with first class honors and $\mathrm{Ph} . \mathrm{D}$. degree in electronic engineering from University of Southampton, UK in 1998 and 2003, respectively. He has been a faculty member at the Multimedia University, Malaysia from 1998 until 2008. He was a visiting fellow at National Institute of Information and Communication Technology (NICT), Yokosuka, Japan in FebruaryMarch, 2005. Since May 2007, he is a senior staff researcher at Wireless Communications Cluster, MIMOS Berhad. His current research interests are cognitive radio and mesh network. He has published over 35 journal and conference papers. He has 18 patents pending at World Intellectual Property Organization (WIPO) and Intellectual Property Corporation of Malaysia (MyIPO).

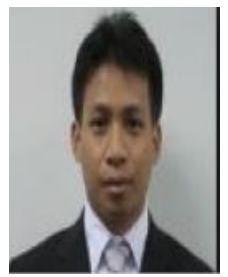

Nordin Ramli obtained his B.Eng, M.Eng and PhD degrees from University of Electro-Communications, Japan. $\mathrm{He}$ is a professional engineer registered under Board of Engineer Malaysia (BEM) and senior member of IEEE. He was a research assistant and teaching assistant at University of ElectroCommunications, Japan at was a lecturer at Multimedia University, Malaysia in year 2008. He was a visiting senior lecturer at University Malaysia Perlis, Malaysia and adjunct lecturer at Open University Malaysia. Now, he is working as staff researcher at MIMOS Berhad, Malaysia. He has lead many research grants and has published more indexed international journals and conferences. He was the chair of Chapter of IEEE Comsoc and Vehicular Technology, Malaysia in year 2013.

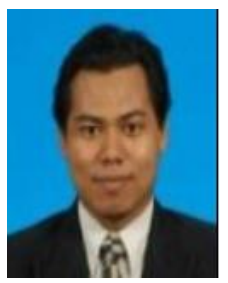

Khairil Anuar received his B.Eng. (Hons) degree in electrical and electronic engineering from University of Salford and masters of science degree in telecommunication from UKM. He was a technical manager at Biztari Technocraft Sdn Bhd in year 2004-2005 and working as a lecturer at Multimedia University since 2005 . His current research interests are wireless mobile communication and data networking. He has won some research competitions including ITEX12 and ITEX13. He is a graduate engineer of Board of Engineer Malaysia and member of IEEE.

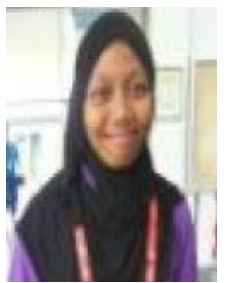

Khairunissa Ismail received the B.Eng. degree in electronic from Multimedia University in 2013. She is working as a research assistant at Multimedia University on cognitive radio networking and further studies in masters of science in Multimedia University. Currently she is working on the algorithm and scheme for spectrum mobility in cognitive radio oriented wireless network sponsored by Science Fund, Ministry of Science and Technology, Malaysia. 